\title{
Zusammenarbeit von Einrichtungen: Eine theoretische Perspektive für die Forschung zum Lebenslangen Lernen
}

\author{
Współpraca między instytucjami. Teoretyczna perspektywa \\ badań nad uczeniem się przez całe życie
}

Streszczenie: Artykuł dotyczy problematyki, która od co najmniej trzech dziesięcioleci zajmuje badaczy systemów oświatowych oraz specjalistów edukacji dorosłych zaangażowanych w rozwój i urzeczywistnienie koncepcji uczenia się przez całe życie. Pytania o inicjatywy, konieczne celem zwiększenia aktywności edukacyjnej obywateli, niezależnie od ich wieku, wciąż na nowo przywołują zagadnienia związane z funkcjonowaniem instytucji edukacyjnych oraz roli różnorodnych partnerów społecznych w procesie kształtowania klimatu i warunków sprzyjających uczeniu się. Uwagę badaczy przykuwa aranżacja środowisk inspirujących procesy uczenia się w społecznościach lokalnych, w regionach, w skali krajowej i międzynarodowej. Dotychczasowe badania nad rolą instytucji w działaniach na rzecz zwiększenia aktywności edukacyjnej dorosłych oraz efektywności procesów uczenia się pokazują, że ich potencjał nie jest w pełni wykorzystany. Ograniczając się do roli oferenta kursów czy innych form edukacyjnych, zaprzepaszczają częstokroć możliwości twórczego i inspirującego kreowania środowisk, w których ludzie pracują, spędzają czas wolny i podejmują różnorodne aktywności, wspierające ich w rozwoju wiedzy i kompetencji. Co jest szczególnie istotne, mimo iż współpraca między instytucjami działającymi w danej społeczności lokalnej lub w pewnym regionie, zdaje się być naturalną konsekwencją sąsiedztwa i dobrze rozumianego interesu społecznego, nie zawsze potencjalni partnerzy wyrażają na nią gotowość. Barierę stanowi obawa przed konkurencją, utratą własnego profilu czy konieczność podporzadkowania się interesom reprezentowanym przez partnerów kooperacji. Artykuł, definiując główne pojęcia związane z instytucją i współpracą między instytucjami (specyfika instytucji, ich profil i zadania oraz funkcje, cele, treści, formy współpracy), ukazuje korzyści wynikające ze współdziałania partnerów społecznych reprezentujących różne obszary życia społecznego, gospodarczego czy kulturalnego (efekt synergii, redukcja kosztów, zwiększenie efektywności działania, rozszerzenie usług i oferty zamiast ich powielania, scalenie kompetencji). Przy- 
wołuje podstawowe założenia wyznaczające istotę kooperacji między instytucjami, z których każda, dążąc do realizacji własnych instytucjonalnych celów, może - dzięki włączeniu się w szersze działania - realizować je z większą skutecznością, przyczyniając się jednocześnie do urzeczywistniania celów ogólnospołecznych, jak choćby ułatwienie dostępu do ofert edukacyjnych i poradnictwa czy podniesienie jakości usług edukacyjnych. Przedstawione zostają m.in. cele i funkcje instytucji i współpracy międzyinstytucjonalnej, efekt synergii wynikający ze współdziałania oraz różnice między współpracą instytucjonalną a działaniem w sieci. $\mathrm{Na}$ podkreślenie zasługuje fakt, że wszystkie obszary instytucjonalne mogą być brane pod uwagę jako potencjalni partnerzy do współpracy z instytucjami edukacyjnymi. Przecież procesy uczenia się zachodzą wszędzie: w fabrykach i biurach, kawiarniach i parkach, we współpracy z gospodarką, kulturą i administracją. Człowiek uczy się wszędzie tam, gdzie napotyka innych ludzi. Artykuł nakreśla również pytania badawcze, istotne w kontekście optymalizacji warunków ramowych uczenia się przez całe życie, zwłaszcza roli, jaka przypada partnerom społecznym, w tym instytucjom oświaty, w procesie kreowania środowisk sprzyjających podejmowaniu aktywności edukacyjnej. Pytania dotyczą m.in. możliwości osiągnięcia efektów synergii poprzez wykorzystanie aktualnego potencjału tkwiącego w regionach i instytucjach; miejsc i kultur nauczania i uczenia się przyjaznych uczeniu się przez całe życie; innowacyjnych rozwiązań na rzecz zwiększenia udziału w uczeniu się osób w każdym wieków i w różnych sytuacjach życiowych, w tym grup społecznych tradycyjnie nie uczestniczących w jakichkolwiek formach edukacji. Urzeczywistnienie koncepcji społeczeństw uczących się przez całe życie implikuje konieczność współpracy możliwie wielu partnerów społecznych: rodzin, podmiotów gospodarczych, stowarzyszeń, instytucji oświaty i kultury. Nie ulega bowiem wątpliwości, że procesy uczenia się w społecznościach lokalnych i w regionach zależą w dużej mierze od relacji łączących wszystkich zainteresowanych aktorów społecznych.

Słowa kluczowe: instytucja; organizacja; współpraca; partnerstwo; sieć; uczenie się przez całe życie.

Summary: The cooperation between institutions remains the traditional topic of adult education. Nowadays, it is gaining new interest among researchers as the popularity of lifelong learning is growing. The cooperation between institutions arises potential which should be used to shape optimal conditions for life-long learning of the society. The article defines the main concepts relating to the cooperation with different social partners. It presents the goals, functions and profits achieved through joint action. Even though some institutions share doubts concerning the cooperation with other organizations on local or regional level, it is essential to notice that cooperation is generally profitable for all involved partners and serves the local community.

Keywords: institution; organization; cooperation; partnership; network; lifelong learning. 


\section{Einführung}

Der Mensch ist ein soziales Wesen. Er konnte in seiner feindlichen Umwelt nur überleben, indem er viele Dinge gemeinsam mit anderen Menschen tat, als Mitglied der Familie, Gruppe, Horde, des Volkes, der Nation. In der Gemeinschaft war es ihm möglich, sich auf die Herausforderungen der Außenwelt einzustellen und die Umwelt zu seinen Gunsten zu verändern. Menschen haben schon immer zusammengearbeitet, um gemeinsame Ziele zu erreichen, und sie werden es immer tun.

Kooperation ist ein heiß diskutiertes Thema in der Verhaltensforschung. Kooperation mit Verwandten - das ist bei Menschen und Tieren zu beobachten. Sogar Verhaltensweisen, die dem Einzelnen Nachteile bringen, setzten sich durch - wenn verwandte Artgenossen profitieren. Aber unter welchen Bedingungen kooperieren Nicht-Verwandte? (Drewes 2010, S. 26).

Auf diese Frage gibt es sehr viele Antworten. So unterschiedlich die Menschen mit ihren biografischen Erfahrungen, Lebens- und beruflichen Lagen, so unterschiedlich sind auch die Beweggründe für die Zusammenarbeit. Für die einen sind es Sympathie für andere Menschen, der Wunsch, etwas Spannendes, Bereicherndes zu erleben oder Neues zu lernen, die Freude am gemeinsamen Lösen von Problemen, das Neugierigsein oder das Bedürfnis nach Zugehörigkeit. Die anderen verbinden mit der Zusammenarbeit die Hoffnung auf Anerkennung, Beschleunigung des Karriereweges oder finanzielle Profite. Wieder andere tun es, weil kein anderer Weg denkbar oder möglich ist, als Kräfte zu bündeln, Kompetenz zu teilen und Wissen zu vereinen. Wandel, Fortschritt und die Entfaltung des Lebens sind auf $\mathrm{Zu}-$ sammenarbeit verschiedener gesellschaftlicher Akteure angewiesen.

Die Bedeutung von interorganisationalen Kooperationen im Bildungsbereich ist unbezweifelbar und kein wirklich neues Thema. Schon in den 1970er-Jahren wurden in westeuropäischen Ländern in bildungspolitischen Dokumenten Empfehlungen zur verbesserten Koordinierung und Effektivitätssteigerung durch Abstimmung zwischen den Einrichtungen auf lokaler und regionaler Ebene formuliert ${ }^{1}$. Die Zusammenarbeit sollte dazu

1 Das Thema „Bildungseinrichtungen“ ist auch in der polnischen Erwachsenenbildung präsent, auch wenn die Aufmerksamkeit der Forscher häufiger der Organisation der Einrichtungen, den Inhalten der Bildung sowie den Lehr - und Lernmethoden als der Kooperation oder Vernetzung gilt, vgl. z. B.: Aleksander 2009; Pleskot-Makulska 1999; Rosalska 2004; Byczkowski, Maliszewski, Przybylska (red.) 2003; Koprowska 2006. 
beitragen, dass Doppelangebote vermieden, angebotsbezogene Überschneidungen abgebaut und unproduktive Konkurrenzen aufgelöst werden (Jütte 2002, S. 13). Mit der Hinwendung zum Lebenslangen Lernen als bildungspolitischem Handlungsziel in den 1990er-Jahren rückten Kooperationen zwischen Weiterbildungseinrichtungen sowie anderen Organisationen in ihrem Umfeld in den Brennpunkt der Aufmerksamkeit (Dollhausen, Feld 2010, S. 24). Die Bündelung der Potenziale soll allen Bürgerinnen und Bürgern die Verwirklichung des Lernens im Lebenslauf ermöglichen und damit ihre persönliche Orientierung, gesellschaftliche Teilhabe und Beschäftigungsfähigkeit verbessern.

Das Konzept des lebenslangen Lernens führt unmittelbar zum Konzept der lernenden Gesellschaft, in der sich eine Vielzahl an Gelegenheiten zum Lernen ergeben, in der Schule wie im wirtschaftlichen, sozialen und kulturellen Leben. Daraus resultiert zwingend die Notwendigkeit, mit den verschiedenen gesellschaftlichen Akteuren, z.B. in Familie, Wirtschaft, Verbänden, Kultur verstärkt und partnerschaftlich zusammenzuarbeiten (UNESCO 1997, S. 134).

Es geht um Stärkung der Autonomie, Eigenverantwortung und Selbststeuerung in den Bildungsinstitutionen, den Unternehmen, bei den Lehrenden und Lernenden sowie Ermöglichung sozialer und kultureller Dynamik, die das Lebenslange Lernen attraktiver macht.

Auch eine andere Tendenz zeigt sich in aller Deutlichkeit: In der globalisierten Welt nimmt die Zusammenarbeit zwischen Regionen, Staaten und Gemeinschaften eine neue Dimension an. Die Globalisierung ist nicht unerwartet aufgetaucht. Bereits seit der zweiten Hälfte des zwanzigsten Jahrhunderts sind wir Zeugen einer ständigen Verdichtung und Intensivierung von Interdependenzen und wirtschaftlichen, ökonomischen, militärischen, politischen und kulturellen Beziehungen. Die Globalisierung wirkt sich auf alle Bereiche der menschlichen Tätigkeit aus: auf das soziale und kulturelle Leben, auf zentrale Werte, Moral und Kunst. Die Globalisierung ist per Definition auf Konsolidierung und auf die Fusion der Potenziale und Bemühungen eines wachsenden Teils der Menschheit ausgerichtet. Einer allgemeinen Überzeugung zufolge kann man mit vereinten Kräften mehr erreichen. Daraus erwachsen die Uniformierung der Welt, neue Formen der Zusammenarbeit, Solidarität und Identität auf der überörtlichen und transnationalen Ebene. Ebenso Organisationen wie die Europäische Union oder die NATO und auch viele Ideen und Konzepte, die uns jeden Tag begleiten: Euro-Regionen, die Zusammenarbeit von Partnerstädten, Netzwerkbil- 
dungen oder auch Programme und Projekte, die die Zusammenarbeit zur Lösung von Problemen, welche mit unterschiedlicher Intensität in lokalen Gemeinschaften, in bestimmten Ländern, Regionen oder Kontinenten auftreten, inspirieren. Als Folge der Globalisierung werden neue Studiengänge in den Bildungsmarkt eingeführt, die mit Bezug auf die Bedürfnisse des europäischen Arbeitsmarktes hin ausbilden. Die Internationalisierung von Studium und Bildungsangeboten wird durch moderne Technologien und eine neue Lernkultur gefördert. Internationale Zusammenarbeit ist freilich mehr als ein Schlagwort unserer Zeit. Sie ist ein Instrument zum Aufbau einer Kultur des Friedens und ein Mittel zur Erhöhung des Lebensstandards aller Menschen. Zugleich ist sie eine große Herausforderung für diejenigen, die ihre Chance, sich am internationalen Geschäft zu beteiligen, noch nicht wahrgenommen haben.

\section{Institutionen und Kooperation}

Der Begriff „Institution“ ist weit gefasst. Meistens definiert sich Institution als ein System von Regeln, die den Individuen, Gruppen oder Gemeinschaften vorgeben, wie sie sich verhalten sollen. „Die institutionale Welt ist vergegenständlichte menschliche Tätigkeit, und jede einzelne Institution ist dies ebenso" (Berger, Luckmann 1991, S. 65). Die von Menschen konstruierten Institutionen prägen die gesellschaftliche Wirklichkeit. Sie schränken die Unsicherheit, Willkür und Beliebigkeit im menschlichen Miteinander ein. Je besser und klarer die Regeln sind, desto effektiver entwickeln sich die Kommunikation, die Zusammenarbeit und alle Bereiche des menschlichen Handelns, z. B. die Wirtschaft. „Je mehr Verhaltensweisen institutionalisiert sind, desto mehr Verhalten wird voraussagbar und kontrollierbar" (Berger, Luckmann 1991, S. 67). In der soziologischen Perspektive versteht man unter Institutionen u. a. die Familie, das Bildungswesen oder die Sprache. Im juristischen Sinne spricht man von rechtlichen Institutionen. Mit einer Rechtseinrichtung ist z.B. das Familienrecht gemeint. Auch „Bildungsprozesse folgen nicht einfach pädagogischen Ideen, sondern bedürfen der Institutionen für ihre Umsetzung" (Leschinsky, Cortina 2005, S. 28).

In der Regel verfügt jedes nationale Bildungssystem über Einrichtungen mit einem direkten oder indirekten Bildungsauftrag. Ebenso unterscheidet ein jedes Bildungswesen im europäischen Raum zwischen öffentlichen Bildungseinrichtungen und Bildungseinrichtungen in freier Trägerschaft. Zu den ersten zählen Kindergärten, Schulen aller Art, Institutionen der Er- 
wachsenenbildung sowie eine Reihe von Einrichtungen mit einem indirekten Bildungsauftrag, z. B. Museen und Bibliotheken. Zu den nicht öffentlichen Bildungseinrichtungen gehören beispielsweise Institutionen in der Trägerschaft von Interessenverbänden, Kirchen, Gewerkschaften, Unternehmen oder privaten Personen. In der Sozialarbeit, Sozialpädagogik und der Weiterbildung werden Institutionen häufig mit Organisationen gleichgesetzt. In diesem Sinne sind einige Forscher der Ansicht: „Betriebe, Einrichtungen, Institutionen und Unternehmen sind allesamt Organisationen“ (Nuissl, Strauch 2008, S. 8). Es existieren viele Definitionen von Organisationen, die jeweils verschiedene Merkmale hervorheben. Als Beispiel hierfür sei genannt:

Eine Organisation ist ein kollektives Ganzes mit relativ festgelegten und identifizierbaren Grenzen, einer normativen Ordnung, hierarchischem Autoritätssystem, Kommunikationssystem und einem koordinativen Mitgliedssystem; dieses kollektive Ganze besteht aus einer relativ kontinuierlichen Basis innerhalb einer sie umgebenden Umwelt und beschäftigt sich mit Handlungen und Aktivitäten, die sich gewöhnlich auf ein Endziel oder Objektiv hin beziehen, oder eine Menge von Endzielen oder Objektiven (Weinert 1992, S. 41).

\section{Zielfunktion der Organisationen}

Die meisten Definitionen von Organisationen betonen ihre Zielfunktion: „Ziele von Organisationen, Einrichtungen, Betrieben definieren einen »SollZustand«, auf den hin die Tätigkeiten in der Organisation gebündelt werden" (Nuissl, Strauch 2008, S. 12). Ziele müssen auf verschiedenen Ebenen formuliert werden. Ihre Aufgabe besteht nicht nur darin, eine allgemeine Handlungsrichtung anzugeben, sondern auch:

1. das Wahrnehmen und Bewerten der Zustände zu beeinflussen („Richtziele“) und damit im normativen Bereich zu steuern,

2. die Mitglieder (Mitarbeiterinnen und Mitarbeiter) zu orientieren, ihnen Kriterien für Handeln und Entscheiden an die Hand zu geben,

3. zu koordinieren, Pläne und organisatorische Regeln in der Organisation aufeinander abzustimmen, eine Kontrolle zu ermöglichen, die umso leichter ist, je präziser und handlungsnäher gesetzte Ziele formuliert sind,

4. die Mitarbeiterinnen und Mitarbeiter zu motivieren, wobei die Erreichbarkeit der Ziele wichtig ist,

5. die Weisungs- und Leitungsmacht zu demonstrieren, 
6. Interessen zu sichern, Freiräume zu wahren und Macht auszuüben sowie

7. Identität und Gemeinsamkeit zu schaffen und zu bewahren (Nuissl, Strauch 2008, S. 12).

Die Zielebene wird unter anderem davon bestimmt, ob die Einrichtung einen profitorientierten oder nicht profitorientierten Charakter hat. Allerdings verliert das Kriterium „kommerzielle“ bzw. „nicht kommerzielle“ Einrichtung seit den 1980er-Jahren an Schärfe.

Der Anteil von Mitteln, die auch in nicht-kommerziellen Bildungseinrichtungen nach marktwirtschaftlichen Prinzipien mit kommerziellen Zielen erwirtschaftet werden, wächst ständig. Es wird in den nächsten Jahren, dies verheißen entsprechende Budgetlinien kommunaler und öffentlicher Haushalte, weiter wachsen. Ehedem als „nicht-kommerzielle Einrichtungen“ gedachte Bildungsorganisationen sind daher mittlerweile längst mindestens janusköpfig: Vielfach wird versucht, mit marktwirtschaftlichen Mitteln nichtkommerzielle Ziele zu verfolgen, einen öffentlichen Auftrag zu erfüllen, der pädagogisch motiviert, aber nicht öffentlich finanziert wird (Nuissl, Strauch 2008, S. 18).

Es wird zunehmend schwieriger, vor allem im Weiterbildungsbereich, idealtypisch zwischen den kommerziellen bzw. nicht kommerziellen Einrichtungen $\mathrm{zu}$ unterscheiden, denn Weiterbildung gehört nicht zu den Bildungssektoren wie Schule oder Hochschule, die überwiegend staatlich finanziert werden. Eine Erfassung der institutionellen Strukturen des Weiterbildungsbereiches ist ein schwieriges Unterfangen (Nuissl, 1999 S. 389 ff.). Dennoch werden verschiedene Dimensionen herangezogen, um das sich stets wandelnde Institutionen- und Angebotsspektrum zu bestimmen. Peter Faulstich zieht als Ordnungskriterien heran: Funktionsbereiche (z.B. allgemeine, berufliche und politische Weiterbildung); inhaltliche Programmschwerpunkte (z.B. sprachliche, technische oder kulturelle Weiterbildung); Adressaten (z.B. Gewerkschaftler, Manager); Angebotsform (offene versus geschlossene Weiterbildung) sowie Rechtsform (z.B. Verein, Anstalt öffentlichen Rechts) (Faulstich 2005, S. 631). Die genannten Kriterien liefern das spezifische Profil der jeweiligen Einrichtung, wobei verstärkt Überschneidungsbereiche entstehen. Wie der Bildungsbereich ständig im Wandel ist, so unterliegen auch die Institutionalisierungsprozesse ihrer eigenen Dynamik, nicht zuletzt infolge eines sich permanent ändernden Nachfrageverhaltens. 


\section{Bildungskooperationen auf regionaler Ebene}

Gegenwärtig stellt Bildung ein zentrales Element der regionalen Zusammenarbeit dar. Die Kooperationen haben eine neue bildungspolitische und mithin institutionelle und praktische Bedeutung erfahren.

insbesondere im Kontext der Umsetzung von Strategien Lebenslangen Lernens gewinnen Kooperationen als zentrale Ansatzpunkte für neue Institutionalformen, von lokalen Bildungszentren bis hin zu regionalen Netzwerken, an Relevanz. Weiterbildungseinrichtungen sind damit vor die Aufgabe gestellt, sich aktiv am Aufbau von kooperativen Bildungsstrukturen zu beteiligen (Dollhausen, Feld 2010, S. 24).

Zum Kooperationsbegriff gibt es viele Synonyme und Definitionen. Als Synonyme stehen für Kooperation Zusammenarbeit, Teamwork, Gruppenarbeit, Partnerschaft. Bezogen auf den Bildungsbereich, verstehen wir unter Kooperation die Zusammenarbeit von mindestens zwei Partnern, die - unter einer gemeinsamen Zielsetzung - bestimmte Aufgabenbereiche übernehmen. Das bedeutet: Kooperationen sind immer arbeitsteilig organisiert, wobei sie zumeist ohne institutionalisierte Führungs- und Moderationsleistungen auskommen (vgl. Kohlmeyer 2001, S. 13; Minderop, Solzbacher 2007, S. 3; Nuissl 2010, S. 26). Kooperationen im Bildungsbereich resultieren oft aus der Notlage, d. h., sie kommen zustande, wenn eine Organisation sich nicht in der Lage sieht, einer bestimmten Herausforderung gerecht zu werden. Für Kooperationen im Bildungsbereich sprechen viele Argumente: die Kostenersparnis, die Arbeitserleichterung, die Kompetenzverschränkung, die Qualitätssteigerung, die bildungspolitische Präsenz, die Zielgruppenerweiterung sowie Innovations- und Entwicklungspotenziale (Nuissl 2010, S. 26-28). Nach Nuissl sind für regionale Bildungskooperationen vier Elemente kennzeichnend: die Ziele, die Partner, die Inhalte und die Formen (Nuissl 2010, S. 28 ff.).

\section{Ziele}

Die Ziele der Kooperation stimmen meistens mit den strategischen Zielen der Einrichtung überein und lassen sich zu drei Komplexen bündeln: Erstens beziehen sich die Ziele auf das aktuelle und zukünftige inhaltliche Spektrum der Einrichtung, z.B.: Eine Institution der politischen Bildungsarbeit geht eine Kooperation mit dem Arbeitslosenverein und dem Sozialhil- 
fezentrum ein, um die bislang unterrepräsentierten, minderqualifizierten und am stärksten von Marginalisierung betroffenen Personengruppen für die Mitbestimmungsmöglichkeiten zu sensibilisieren und ihre politische Partizipation zu erhöhen. Der zweite Zielkomplex betrifft die erhöhte Effizienz der eigenen Arbeit, die Kostenreduktion sowie Synergieeffekte, die über Kooperationen erreicht werden. Z.B., die in einer Region tätigen Weiterbildungsinstitutionen treffen Kooperationsvereinbarungen, um gemeinsam für eine größere Weiterbildungsbeteiligung der Bevölkerung zu werben und damit ihre eigene Reichweite zu erhöhen. Der dritte Komplex erstreckt sich auf die Professionalität und Qualität der Arbeit im weitesten Sinne. Als Beispiel sei hier eine Kooperation zwischen Weiterbildungseinrichtungen und der Wirtschaft in Fortbildung der Lehrkräfte genannt. Sie ermöglicht den Know-how-Transfer aus der Wirtschaft in die Weiterbildungspraxis, gibt Impulse für gute, den Anforderungen des modernen Arbeitsmarktes angemessene Bildung, und erhöht die Qualität der pädagogischen Arbeit.

\section{Partner der Bildungskooperation}

In jeder Region agieren verschiedene Institutionen. Es sind Kindergärten, Schulen unterschiedlichen Typs, Weiterbildungseinrichtungen, Hochschulen und Universitäten, aber auch andere Einrichtungen, wie Museen, Theater, Gedenkstätten, Bibliotheken, die einen Bildungsauftrag haben. In der Fachliteratur werden drei Typen von Kooperationen unterschieden: Erstens sind es typidentische Partnerschaften: Hier finden sich Einrichtungen gleichen Typs zusammen, z. B. eine Hochschule kooperiert mit einer anderen Hochschule auf der Basis eines gemeinsamen Konzepts. Die Grundlage für die Kooperation bildet in diesem Fall das gemeinsame Interesse an der Lösung eines Problems. Die Vorteile sind offensichtlich: Die Konkurrenz wird vermieden, und Synergieeffekte können erzeugt werden. Zweitens handelt es sich um typübergreifende Kooperationen: Hierzu gehören Partnerschaften zwischen Einrichtungen des eng verstandenen Bildungsbereiches, z. B. Kooperationen zwischen Schule und Weiterbildung oder Weiterbildung und Hochschule. Drittens sind es bildungsimmanente Kooperationen: Hierzu zählen Einrichtungen unterschiedlichster Art, die zum Zwecke der Bildung zusammenarbeiten, z. B. Zusammenarbeit zwischen Schulen und Gedenkstätten oder politischen Vereinen (Nuissl 2010, S. 30-31).

Alle institutionellen Bereiche kommen für eine Kooperation mit Bildungseinrichtungen infrage, denn Bildung findet überall statt: am Arbeitsplatz, in Werkstätten und Büros, im Ehrenamt, auf Reisen, in Freizeitparks, 
in der Zusammenarbeit von Wirtschaft und Staat. Gelernt wird, wo auch immer Menschen einander begegnen.

\section{Inhalte der Bildungskooperation}

Zu den häufigsten Kooperationsschwerpunkten zählen nach Wolfgang Jütte: gegenseitige Information, gemeinsame Werbung und Ankündigung von Veranstaltungen, Vermittlung von Teilnehmern, Erfahrungsaustausch, Erschließung neuer Aufgabenfelder, Vermittlung von Dozenten, gemeinsame Projekte, Veranstaltungen, Bildungsangebote, Tagungen, Austausch von Mitarbeitern und Kursleitern, Finanzierung bzw. Kofinanzierung einer Veranstaltung, Mitarbeit in Arbeitskreisen, gemeinsame Interessenvertretung gegenüber Dritten, Programmabsprachen, Nutzung von Gebäuden und Geräten, Fortbildung von Mitarbeitern und Kursleitern, Bedarfsanalysen, Moderation (Jütte 2002, S. 60). In der Regel entstehen Kooperationen auf der Basis des gemeinsamen Interesses und gleicher bzw. ähnlicher Zielsetzung. Eine der Definitionen betrachtet Kooperation als ein Verfahren, „bei dem im Hinblick auf geteilte oder sich überschneidende Zielsetzungen durch Abstimmung der Beteiligten eine Optimierung von Handlungsabläufen oder eine Erhöhung der Handlungsfähigkeit bzw. Problemlösungskompetenz angestrebt wird“ (v. Santen, Seckinger 2003, S. 29). Kooperation bezeichnet eine freiwillige Zusammenarbeit zweier oder mehrerer Partner, die eine gemeinsame Zielstellung vertreten, ihre Aufgaben arbeitsteilig erfüllen und in einem Prozess ein neues positives Ergebnis anstreben.

\section{Netzwerke}

Kooperation und Vernetzung in der Weiterbildung haben Konjunktur, obwohl sie eher zu den alten Themen gehören. Kooperationen können in Netzwerke übergehen, d. h., sie können eine „logisch höher stufige Form sozialer Strukturierung" (Schäffter 2004, S. 33) annehmen. Mit einem Netzwerk haben wir zu tun, wenn reziproke und kooperative Beziehungen zwischen mehreren, aber mindestens drei rechtlich autonomen Akteuren vorliegen, die von relativer Dauer sind (Sydow 1992, S. 79 ff.). Netzwerke und Kooperationen liegen dicht beieinander, doch es gibt Merkmale, die die beiden Formen der Zusammenarbeit unterscheiden. 
Tab. 1. Differenzen zwischen Kooperation und Netzwerk

\begin{tabular}{|l|l|l|}
\hline \multicolumn{1}{|c|}{ Merkmal } & \multicolumn{1}{|c|}{ Kooperationen } & \multicolumn{1}{c|}{ Netzwerke } \\
\hline Größe & $\begin{array}{l}\text { Beteiligung von zwei oder mehreren } \\
\text { Partnern und/oder Einrichtungen, } \\
\text { aber in der Regel ist die Zahl der } \\
\text { Beteiligten geringer als in Netz- } \\
\text { werken. }\end{array}$ & $\begin{array}{l}\text { Beteiligt sind prinzipiell mehr Partner } \\
\text { und Einrichtungen als an einer } \\
\text { Kooperation. }\end{array}$ \\
\hline Zielvorgabe & $\begin{array}{l}\text { Es gibt ein definiertes konkret } \\
\text { vereinbartes Ziel. }\end{array}$ & $\begin{array}{l}\text { Offenheit, Flexibilität und Dynamik bei } \\
\text { der Formulierung der Ziele. }\end{array}$ \\
\hline Kommunikation & $\begin{array}{l}\text { Es wird direkt zwischen zwei oder } \\
\text { drei Partnern verhandelt. }\end{array}$ & $\begin{array}{l}\text { Gruppenberatungen, die in bi- oder } \\
\text { trilateralen Gesprächen vor- und } \\
\text { nachbereitet werden. }\end{array}$ \\
\hline Kooperationsstruktur & $\begin{array}{l}\text { Direkte Steuerung und Über- } \\
\text { wachung der Kommunikation }\end{array}$ & $\begin{array}{l}\text { Partnerübergreifende Steuerung } \\
\text { (Netzwerkmanagement) }\end{array}$ \\
\hline
\end{tabular}

Quelle: eigene Darstellung in Anlehnung an Nuissl 2010, S. 77.

Claudia Solzbacher und Dorothea Minderop definieren den Begriff des Bildungsnetzwerkes folgendermaßen:

Bildungsnetzwerke bündeln Kompetenzen und Ressourcen, um gemeinsame Ziele zu erreichen, mit denen jeder Partner für sich allein überfordert wäre. Sie sind in der Regel verbindlicher organisiert und gesteuert als Kooperationen. Die Partner stellen ihre jeweilige Autonomie jedoch nicht in Frage. Es geht vielmehr darum, den Transfer von Kenntnissen und Kompetenzen zu ermöglichen und zu verbessern - dies macht die Idee des Netzwerkens heute besonders aktuell -, die Kosten zu senken, indem Synergien genutzt werden (Solzbacher, Minderop 2007, S. 3).

Am Rande bemerkt: Bereits Anfang des 20. Jahrhunderts haben die Sozialwissenschaftler Georg Simmel und Norbert Elias auf die Verflechtung zwischen Individuum und Gesellschaft hingewiesen und Netzwerke als die Basis des sozialen Lebens überhaupt verstanden. Die Gesellschaft galt schon damals als eine "Netzwerkgesellschaft" (Weber 2002, S. 10). Gegenwärtig werden Netzwerke zunehmend als eine zukunftsträchtige vielversprechende Strategie zur Bewältigung gesellschaftlicher Probleme gesehen. Zu ihren Stärken gehört, dass sie auf dem Konsensus vieler verschiedener Akteure bezüglich der Ziele des gemeinsamen Handelns beruhen. Entscheidend sind die Zusammensetzung und das Potenzial der Partner. In erster Linie geht es 
doch um die Kompetenzbündelung, den Transfer von Kenntnissen und die Fähigkeit zu kommunizieren. „Die Vorteile von Netzwerken liegen zweifellos in ihren offenen, beweglichen und auf Freiwilligkeit basierenden Strukturen" (Nuissl 2010, S. 78). In der Regel sind Netzwerke für alle bestehenden und neuen Kooperationen offen. Am wichtigsten ist jedoch die Fähigkeit eines funktionsfähigen Netzwerkes, Synergien zu schaffen, die Lebens- und Strategiefähigkeit sowie das Durchsetzungspotenzial der Akteure zu erhöhen (Bullinger, Nowak 1998, S. 12 ff.). Im Zusammenhang mit Kooperation und Netzwerkarbeit im Bildungsbereich bezeichnen Synergieeffekte den erwarteten Nutzen, der sich infolge der Zusammenarbeit ergibt. Die wichtigste Voraussetzung für das Erzielen der Synergieeffekte bildet die Zustimmung und Bereitschaft aller Beteiligten zur Zusammenarbeit. Nur wenn die Mitarbeitenden die neue Arbeitsform akzeptieren und unterstützen, stehen Nutzen und Erfolge in Aussicht. Im Zusammenhang mit internationaler Kooperation und Netzwerkarbeit sind auch noch andere Synergieeffekte zu betonen. Hier handelt es sich vorwiegend um die Chance, spezifisches Wissen und Kenntnisse ausländischer Partner einzubeziehen, neue Blickwinkel zu gewinnen und kreative Handlungsalternativen zu erkennen. Internationale Kontakte regen die Entwicklung neuer Ideen und Lösungen an, tragen zur Klärung eigener (nationaler) Probleme bei und geben Rückenstärkung und Motivation.

Es sei noch kurz darauf hingewiesen, dass je nach Kooperation oder Vernetzung unterschiedliche Probleme auftreten können. Zu den häufigsten kritischen Punkten gehören nach Nuissl: Konflikte wegen Unklarheiten (z. B. wenn Vereinbarungen nicht genug präzise Formulierungen enthalten), Interessenkonflikte (z.B. auf der Basis neuer institutioneller Tatbestände bei den Partnern), Konflikte wegen eines nicht zufriedenstellendes KostenNutzen-Verhältnisses, Kommunikationsprobleme sowie Probleme in Folge von Rivalität oder Veränderungen der Rahmenbedingungen (Nuissl 2010, S.41-44). Die meisten Probleme lassen sich jedoch beheben, vorausgesetzt dass Motivation, Wille und Bereitschaft zur Zusammenarbeit bei den Partnern vorhanden sind.

\section{Zusammenfassung}

Es besteht ein großer Forschungsbedarf im Bereich Bildungseinrichtungen im sozialen und räumlichen Kontext. Lebenslanges Lernen erfordert Unterstützung, Anleitung, Coaching sowie Beratung der Lerner, unabhän- 
gig davon an welchem Lernort -Schule, Betrieb, Hochschule, Weiterbildungseinrichtung oder im Privatleben - sie lernen. Daher müssen geeignete Rahmenbedingungen und Systemvoraussetzungen geschaffen werden, die auf lebenslanges Lernen ausgerichtet sind, und neue Lernkulturen sowie Innovationen effektiv auf den Weg bringen. Lebenslanges Lernen für alle stellt eine Querschnittsaufgabe aller Bildungsbereiche dar und muss auch im Zusammenhang mit regionalen- und Lebensbesonderheiten der Bürgerinnen und Bürger behandelt werden. Es ist bekannt, dass regionale Lernprozesse maßgeblich von Relationen zwischen einzelnen Akteuren abhängen, davon in welchem Maße und welche Inhalte sie untereinander kommunizieren (Dobischat u.a. 2008, S. 33). Der Erfolg des Konzepts Lebenslanges Lernen bedarf einer Weiterentwicklung der Strukturen, der Bildungsinhalte, der Vermittlungs- und Lernformen sowie der Erhöhung der Bildungsbereitschaft möglichst vieler Bürgerinnen und Bürger. Die Kooperation aller Bildungsbereiche und verschiedener gesellschaftlicher Akteure schafft hierfür die notwendigen Voraussetzungen. Die gegenwärtig für die Wissenschaft wichtigsten Forschungsfragen sind: Wie können vorhandene Potenziale genutzt werden, um Synergieeffekte zur Bewältigung der Komplexität des Lebenslangen Lernens zu erreichen? Welche Lernorte, Lernarrangements, Lehr- und Lernkulturen unterstützen das Lebenslange Lernen? Wie kann die Kooperation verschiedener Partner die Durchlässigkeit zwischen den Bildungsbereichen erhöhen und die Bildungsbereitschaft der Menschen in allen Lebenslagen- und -phasen fördern? Wie kann erreicht werden, dass auch bildungsferne Gruppen (Weiter-) Bildungsangebote wahrnehmen? Damit ist die Liste der Forschungsfragen aber noch längst nicht erschöpft. Mittlerweile nimmt das Interesse der Wissenschaft an Optimierung der Rahmenbedingungen des Lebenslangen Lernens in der Weiterbildung rasant zu. Nicht zuletzt wird es durch konkrete Erfahrungskontexte und Praxiszusammenhänge geprägt.

Die Zusammenarbeit im Bildungsbereich zwischen verschiedenen gesellschaftlichen Partnern gewinnt zunehmend an Dynamik. Sie wird immer lebendiger, intensiver und bringt zusätzliche greifbare Vorteile auf der lokalen, nationalen und internationalen Ebene. Insbesondere die internationale Zusammenarbeit wächst kraftvoll infolge der Programme der Europäischen Kommission, die konsequent die Idee der Zusammenarbeit über Ländergrenzen hinweg fördert. Die Verflechtung lokaler Prozesse und die globale Verankerung der Probleme der Gegenwart führen zur Intensivierung weltweiter Beziehungen auf zahlreichen Ebenen. Institutionen der Politik, der Wirtschaft und der Bildung arbeiten weltweit vernetzt auf der Basis 
internationaler Kooperationen und Projekte, die die Leistung und Produktivität erhöhen. In der Forschung z.B. schaffen sie einen wissenschaftlichen Mehrwert, welcher in manchen Forschungsbereichen ohne die Bündelung des Wissens, der Kompetenzen und anderer, auch finanzieller Ressourcen unerreichbar wäre. Es ist heute selbstverständlich, dass Forschungsinstitute, Universitäten, wirtschaftliche Unternehmen und sonstige Institutionen spezialisierten internationalen Netzwerken angehören. Die Vorteile der Zusammenarbeit im Bildungsbereich liegen auf der Hand. Zu den wichtigsten zählt der Synergieeffekt, der auf einer Vervielfachung jener Vorteile beruht, die dank der geschickten Kombination von Potenzialen, Kompetenzen, Kenntnissen und Erfahrungen der verschiedenen Partner gewonnen werden können. Genauso wichtig ist der kognitive Wert, der aus der Zusammenarbeit mit Menschen, die andere Sprachen sprechen und verschiedenen Kulturkreisen entstammen, entsteht. Die Arbeit an der Lösung eines gemeinsamen Problems bringt Menschen näher zusammen und baut gegenseitigen Respekt und Verständnis auf. Sie bietet die Möglichkeit, andere Kulturen, Bräuche, Architektur, aber auch die Engel und Dämonen anderer Länder kennenzulernen. Sie macht die Menschen, die an ihr teilnehmen, geistig reicher, emotional empfindsamer und offener für Menschen und ihre Probleme. Sie entwickelt alle Kräfte, die in der Arbeitswelt und dem gesellschaftlichen Leben der modernen Europäer zählen.

Bildung vollzieht sich (...) in institutionellen Zusammenhängen, die sich in einem längeren historischen Prozess entwickelt haben, der in Industrienationen deutlich von der ökonomischen und der damit zusammenhängenden sozialen Entwicklung geprägt ist. Von der Struktur her ähneln sich daher Bildungssysteme moderner Gesellschaften, sodass sich national-spezifische Lösungsvarianten vielfach auf vergleichbare Problemlagen beziehen (Leschinsky, Cortina 2005, S. 28).

Schließlich sollten an einer Zusammenarbeit interessierte Akteure bedenken, dass das Nutzenkriterium nicht außer Acht gelassen werden darf. Eine Zusammenarbeit ist dann fruchtbar, wenn sich daraus für alle beteiligten Seiten Vorteile ergeben.

\section{Literatur}

Aleksander T. (2009), Andragogika, Instytut Technologii Eksploatacji - PIB, Radom, Kraków. 
Berger P. L., Luckmann T. (1991), Die gesellschaftliche Konstruktion der Wirklichkeit. Eine Theorie der Wissenssoziologie, Fischer Taschenbuch Verlag, Frankfurt a. Main.

Bullinger H., Nowak J. (1998), Soziale Netzwerkarbeit. Eine Einführung für soziale Berufe, Lambertus Verlag, Freiburg im Breisgau.

Byczkowski M., Maliszewski T., Przybylska E. (Hrsg.) (2003), Uniwersytet Ludowyszkoła dla życia, Kaszubski Uniwersytet Ludowy, Wieżyca.

Carlsen A. (2013), Networks and networking in adult education as means of learning and addresseing chalenges in the Nordic region, „Rocznik Andragogiczny“, S. 331-342.

Dobischat, R., Düsseldorff Ch., Nuissl E., Stuhldreier J. (2008), Lernende Regionen - begriffliche Grundlagen, [in:] E. Nuissl, R. Dobischat, K. Hagen, R. Tippelt (Hrsg.), Regionale Bildungsnetze, W. Bertelsmann Verlag, Hamburg, S. 23-33.

Dollhausen K., Feld T. C. (2010), Entwicklungslinien und Perspektiven für Kooperationen in der Weiterbildung. Für Lebenslanges Lernen kooperieren, „DIE Zeitschrift für Erwachsenenbildung“, H. 1, S. 24-26.

Drewes E. (2010), Wie Du mir, so ich Dir. Kooperation bei Menschen und Tieren, „DIE Zeitschrift für Erwachsenenbildung“, H. 1, S. 26-27.

Faulstich P. (2005), Weiterbildung, [in:] K. S. Cortina, J. Baumert, A. Leschinsky, K. U. Mayer, L. Trommer (Hrsg.), Das Bildungswesen in der Bundesrepublik Deutschland. Strukturen und Entwicklungen im Überblick, Rowohlt Taschenbuch Verlag, Reinbek bei Hamburg, S. 625-659.

Jütte W. (2002), Soziales System Weiterbildung, W. Bertelsmann Verlag, Bielefeld.

Kohlmeyer K. (2001), Netzwerke in der Jugendberufshilfe. Gestaltungsmöglichkeiten lokaler Kooperation, Institut für berufliche Bildung und Weiterbildung e. V., Göttingen.

Koprowska D. (2006), Założenia i efekty funkcjonowania ośrodków ustawicznej edukacji zawodowej, IBE, Warszawa.

Leschinsky A., Cortina K. S. (2005), Zur sozialen Einbettung bildungspolitischer Trends in der Bundesrepublik, [in:] K. S. Cortina, J. Baumert, A. Leschinsky, K. U. Mayer, L. Trommer (Hrsg.), Das Bildungswesen in der Bundesrepublik Deutschland. Strukturen und Entwicklungen im Überblick, Rowohlt Taschenbuch Verlag, Reinbek bei Hamburg, S. 20-51.

Nuissl E. (1999), Ordnungsgrundsätze der Erwachsenenbildung in Deutschland, [in:] R. Tippelt (Hrsg.), Handbuch Erwachsenenbildung/Weiterbildung, Leske + Budrich, Opladen, S. 389-401.

Nuissl E. (2010), Netzwerkbildung und Regionalentwicklung, Waxmann, Münster, New York.

Nuissl E., Strauch A. (2008), Leiten von Weiterbildungseinrichtungen, Technische Universität Kaiserslautern.

Pleskot-Makulska K. (1999), Szkolnictwo ogólnokształcace i zawodowe dla dorosłych w Polsce w latach dziewięćdziesiątych, „Rocznik Andragogiczny“, S. 95-110. 
Rosalska M. (2004), Katolickie uniwersytety ludowe wobec zmian współczesnej wsi polskiej, Garmond Oficyna Wydawnicza, Poznań.

Schäffter O. (2004), Auf dem Weg zum Lernen in Netzwerken - Institutionelle Voraussetzungen für lebensbegleitendes Lernen, [in:] R. Brödel (Hrsg.), Weiterbildung als Netzwerk des Lernens: Differenzierung der Erwachsenenbildung, W. Bertelsmann Verlag, Bielefeld, S. 29-48.

Solzbacher C., Minderop D. (Hrsg.) (2007), Bildungsnetzwerke und Regionale Bildungslandschaften. Ziele und Konzepte, Aufgaben und Prozesse, Luchterhand, München.

Stopińska-Pająk A. (2015), Polskie tradycje instytucji edukacji dorostych w kontekście uczenia się całożyciowego, „Rocznik Andragogiczny”, S. 289-300.

Sydow J. (1992), Strategische Netzwerke: Evolution und Organisation, Gabler, Wiesbaden.

UNESCO (1997), Lernfähigkeit. Unser verborgener Reichtum, UNESCO-Bericht zur Bildung für das 21. Jahrhundert, Luchterhand, Neuwied.

v. Santen E., Seckinger M. (2003), Kooperation: Mythos und Realität einer Praxis. Eine empirische Studie zur interinstitutionellen Zusammenarbeit am Beispiel der Kinder- und Jugendhilfe, Verlag Deutsches Jugendinstitut, München.

Weber S. (2002), Netzwerk - Interventionen - Vielfalt in Organisationen und Organisationsnetzwerken, [in:] Vernetzungsprozesse gestalten. Erfahrungen aus der Beraterpraxis mit Großgruppen und Organisationen, Gabler, Wiesbaden, S. 9-37.

Weinert A. B. (1992), Lehrbuch der Organisationspsychologie, Beltz Verlag, Weinheim, Basel. 